\title{
La "Quinta Disciplina” en el contexto del proyecto social de la Modernidad
}

\author{
Suárez L. Roldán*
}

\section{Resumen}

El propósito del presente artículo es doble. En primer lugar se pretende destacar un aspecto de la "Quinta Disciplina" que suele pasar desapercibido. Se trata de que en ese discurso podemos encontrar elementos que indican la presencia de un proyecto social a gran escala que trasciende lo que, tradicionalmente, era considerado el ámbito de lo organizacional. En segundo lugar, se pretende mostrar que este proyecto social se aleja drásticamente del proyecto social propio de la modernidad (representado aquí por el discurso filosófico de la llustración). El artículo se inicia con una indagación en la "esencia" del cambio promovido por la "Quinta Disciplina". Esta "esencia" es identificada con un cierto modo de pensar la plenitud del ser humano que dibuja una relación inédita, absolutamente armónica, entre el individuo y la organización. Se muestra cómo este cambio implica un nuevo modo de organización de la sociedad en su conjunto. Finalmente, se muestra cómo este cambio, en sus distintos niveles, y a pesar de hacer uso de nociones modernas como sus vehiculos, rompe por completo con el proyecto de la llustración.

Palabras clave: Quinta Disciplina, organización inteligente, pensamiento sistémico, libertad, Modernidad.

Recibido: 98-05-17 . Aceptado: 98-07-13

* Ingeniero Electrónico de la Universidad de Minería y Metalurgia "Stanislaw Staszic" (Cracovia, Polonia), Maestría en Sistemología Interpretativa de la Universidad de los Andes (Mérida, Venezuela). Profesor del Departamento de Sistemología Interpretativa de la ULA; realizando estudios de doctorado en esta misma área. Dirección: Escuela de Sistemas, Fac. de Ingenieria, Universidad de los Andes, Mérida, Venezuela. 


\section{The "Fifth Discipline" in the Context of the Modern Social Project}

\section{Abstract}

This article has a double purpose. In the first place, it attempts to distinguish an aspect of the "Fifth Discipline" which is normally overlooked. We will try to find elements in this discourse which indicate the presence of a large scale social project which transcends what traditionally was considered the organizational environment. In the second place, we will try to show that this social project is drastically different from the modern social project (represented here by the philosophical discourse of illustration). The article begins with a questioning of the "essence" of change promoted by the "Fifth Discipline". This "essence" is identified with a certain way of thinking of the fullness of humanity presented in the image of a ideal, absolutely harmonious relationship between the individual and the organization. We show that this change implies a new manner of total social organization. Finally we show how this change, in its different levels, even when it employs modern notions in its conveyance, completely breaks away from the project of illustration.

Key words: Fifth discipline, intelligent organization, systematic thought, liberty, modernism.

\section{Introducción: sistemas humanos autodiseñados}

En las últimas décadas, en el campo de las Ciencias de Gerencia y Administración, han aparecido distintas publicaciones que pretenden una humanización de las formas modernas de organización del trabajo. Entre ellas podemos citar, por ejemplo: Calidad Total (Deming, 1993), Reingeniería (Hammer y Champy, 1993), Justo a Tiempo (Majima, 1994), etc. Esta humanización pretende dibujarse en contra de las maneras inhumanas de funcionamiento de las organizaciones productivas -maneras provenientes de la tradición del "salvaje" capitalismo decimonónico- en particular, contra la alie- nante "mecanización" de las tareas y la subsiguiente "robotización" del trabajador. El trabajador -argumentan los nuevos humanistas- no era tratado antes como una persona: su dignidad era veja$\mathrm{da}$, sus aspiraciones personales no eran tomadas en cuenta, a sus disposiciones intelectuales naturales no les era dado desarrollarse a plenitud. Como vemos, este coincide, en general, con el análisis marxista: el trabajador tradicionalmente era tratado como un simple objeto, lo cual le impedia autorrealizarse como ser humano mediante su trabajo.

Los nuevos humanistas argumentan, también, que tal estado de cosas, heredado de épocas pasadas, trae actualmente otro tipo de repercusiones negati- 
vas. Por un lado, la cosificación del trabajador es fuente potencial de conflictos en la sociedad moderna; conflictos que perturban el sistema económico e impiden un armonioso y sostenido aumento del nivel de bienestar de toda la población. Por el otro, los procesos productivos organizacionales, en general, no logran alcanzar el grado de eficiencia óptimo-que pudiera lograrse en caso de que todos los interesados participasen, activamente, con su espíritu creativo e innovador, en el mejoramiento de la eficiencia, efectividad y calidad de la producción.

La solución a estos problemas parece ser, entonces, muy sencilla: hace falta un cambio radical, una gran revolución en las formas en las que han venido funcionando las organizaciones tradicionales. Frente a la autoritaria y burocrática organización jerárquica de férreo control centralizado, hay que proponer una organización democrática que abra un espacio de libertad y autorrealización para cada uno de sus miembros, que permita una participación creativa en la definición de fines comunes y en el continuo mejoramiento de la eficiencia en el logro de esos fines. Una organización que, a fin de cuentas, se abra a la participación de todos sus miembros en la toma de decisiones concernientes a la organización. Un típo de organización como ese permitiría solucionar los tres grandes problemas -mencionados anteriormente- que plantea el funcionamiento de las organizaciones tradicionales y permitiria, finalmente, alcanzar un alto nivel de bienestar social y de felicidad. La gran idea de estas nuevas y revolucionarias organizaciones podríamos resumirla, entonces, en la expresión "Sistemas Humanos Autodiseñados".

La pregunta que quisiera abordar en este artículo es si pudiera pensarse este fenómeno del nuevo humanismo como un renacimiento del proyecto social de la era moderna -al que le son tan caros los ideales de libertad, justicia y democracia. Intentaré, pues, penetrar en la esencia del cambio organizacional que propone uno de los más recientes discursos del nuevo humanismo, para proceder, luego, a comparar su planteamiento con el que le es propio al proyecto de la llustración. Nótese que tal modo de entocar esta corriente gerencial supone que todos estos discursos son más que simples "modas", como lo sugiere Jackson (1993). Se trata de investigar un posible papel social que pueden estar jugando estos discursos y que va más allá del propósito exclusivo de "afinar" el funcionamiento de las organizaciones.

\section{La "Quinta Disciplina": diseño de "organizaciones inteligentes"}

Uno de los discursos más acabados $y$ coherentes en esta corriente del nuevo humanismo es, a mi manera de ver, el que presenta Peter Senge en su "Quinta Disciplina". Revisemos, resumidamente, sus planteamientos principales.

\section{El principio: la Quinta Disciplina}

La manera en la que Senge hace concreta la idea de un Sistema Humano Autodiseñado es a través de la noción de "Organización Inteligente" como organizaciones donde la gente expande conti- 
nuamente su aptitud para crear los resultados que desea, donde se cultivan nuevos y expansivos patrones de pensamiento, donde la aspiración colectiva queda en libertad, y donde la gente aprende continuamente a aprender en conjunto" (Senge, 1990: 11).

Las nociones de "inteligencia" y "aprendizaje" parecen ser, a lo largo de todo su discurso, conceptos claves para Senge. Como veremos, estas nociones están intimamente ligadas a su procedencia intelectual del movimiento -como él mismo lo llama- del "pensamiento sistémico". Tratemos de entender, por ahora, qué significan estos conceptos y por qué son tan importantes.

Inteligencia es capacidad para el aprendizaje. Pero, ¿qué significa aprendizaje? Aprendizaje es -como ya lo sugiere Senge en la cita anterior-el proceso mediante el cual se maximiza la "aptitud para crear los resultados que se desea". Aprender es, entonces, adquirir capacidad para actuar efectivamente de acuerdo a ciertos fines preestablecidos.

Senge afirma que los problemas históricos de la organización social $-y$, en particular los referentes a la organización de la producción- tienen su raiz en una gran falla en los procesos de aprendizaje. En efecto, tanto los conflictos políticos y sociales, como los problemas ecológicos, económicos y organizacionales $e$, incluso, los conflictos estrictamente personales, son resultado de una forma fallida, incompleta de entender el mundo que impide un aprendizaje efectivo. Todos estos problemas son síntomas de que no hemos aprendido a actuar efectivamente como individuos y como sociedad. No será posible lograr, a ninguno de estos niveles, los resultados que deseamos mientras no tomemos conciencia de esta situación.

Esta forma incompleta de ver el mundo la llama Senge "pensamiento asistémico". El pensador asistémico puede ser caracterizado de la siguiente manera:

a) Puede ver conexiones sólo entre eventos muy poco separados en el espacio y en el tiempo.

b) Por tanto concibe el mundo en términos de procesos lineales.

c) De aquí que no pueda darse cuenta de que él mismo es causa de muchos eventos y situaciones que percibe como fortuitos.

Estas son las razones por las cuales el pensador asistémico no puede aprender a influir teleológicamente sobre procesos de gran complejidad: al no percibir, como tales, las consecuencias de muchas de sus acciones, no se puede dar en él el proceso natural de aprendizaje por ensayo y error. La gran panacea es, entonces, el "pensamiento sistémico". El pensador sistémico está consciente de la existencia de procesos muy complejos (grandes redes causa-efecto) que continuamente se están dando a nuestro alrededor $\mathrm{e}$ influyen invisiblemente sobre nuestras vidas. Sabe que nosotros mismos formamos parte de ellos y que, para descubrirlos, es necesario poner atención a eventos a veces muy distanciados en el espacio y en el tiempo. Asi, el pensamiento sistémico -o, como lo llama Senge, la Quinta Disciplina-se convierte en el principio más fundamental de una forma mucho más efectiva de aprendizaje que permitirá, finalmente, alcanzar 
efectivamente nuestras metas individuales y sociales.

\section{Practicando el principlo:}

\section{las 4 disciplinas}

Una Organización Inteligente debe ser un ente colectivo tan coherente que actúe como un sólo ser inteligente. Esta es la necesidad fundamental, el objetivo que se deriva de la demanda de acción colectiva efectiva. La inteligencia de este ser no puede estar centralizada y acumulada sólo en un "puesto de mando", como sucedia en las organizaciones tradicionales. La complejidad de las organizaciones modernas requiere una inteligencia y un control "dispersos" a lo largo y ancho de toda la organización. Pero ¿cómo conservar la coherencia de acción dispersando, al mismo tiempo, la inteligencia y el control? Esto requiere de cuatro disciplinas adicionales:

1. La construcción de una visión (o imagen) clara y concreta-tanto del futuro organizacional deseado, como del modo de funcionamiento actual de la organización-que pueda ser aceptada y compartida, como fin común, por todos sus miembros. Esta visión permitirá mantener la necesaria unidad de sentido de la acción organizacional. Este es el punto atacado por la disciplina "Visión Compartida".

2. La construcción de equipos que sepan cooperar y comunicarse coherentemente, donde las acciones de todos sus miembros se refuercen mutuamente, o sea, logren un "alineamiento". La disciplina que se enfoca en este punto es la de "Aprendizaje en Equipo".
3. Los puntos anteriores requieren de la asimilación, a nivel individual, de nuevas herramientas que agudicen el pensamiento sistémico. Además de los arquetipos de la "Quinta Disciplina", está la disciplina de los "Modelos Mentales" que permite revisar continuamente nuestras micro-teorías sobre los procesos que se dan en la realidad.

4. Finalmente, además de las aptitudes anteriores, se requiere de un cambio fundamental de actitud de todos los miembros de la organización. De este punto se ocupa la disciplina del "Dominio Personal".

Ahora bien; enfoquémonos en este último punto que muestra cierta singularidad: mientras los primeros tres puntos se ocupan de desarrollar herramientas o habilidades, el "Dominio Personal" pretende algo en apariencia más profundo, una nueva actitud. Más aún, esta actitud es, simultáneamente, condición de posibilidad y objetivo final de las 5 disciplinas. Veamos esto más de cerca.

En efecto, Senge, por un lado, parece llegar a la conclusión de que, a fin de cuentas, el éxito del proceso de diseño de una Organización Inteligente descansa sobre una cierta actitud que se asume voluntariamente o no se asume: "[... ] Sólo a través de la opción un individuo llega a practicar las disciplinas del aprendizaje. Estar en un ámbito alentador es una ayu$\mathrm{da}$, pero no elimina la necesidad de opción. Las organizaciones inteligentes sólo pueden ser construidas por individuos que ponen su espiritu vital en la tarea. Nuestras opciones guían ese espiritu" (Senge, 1990: 444, subrayado mio). 
Por otra parte, la implementación de las disciplinas en una organización, según Senge, debe darse en el mismo orden que fue descrito arriba, es decir, que el "Dominio Personal" es la última disciplina en aplicarse. Es, además, la disciplina que define el "ser" más íntimo de los miembros de una Organización Inteligente -de hecho, la disciplina del "Dominio Personal" es llamada por Senge "el espiritu de la Organización Inteligente". Pero, ¿cómo es posible este doble papel del "Dominio Personal", de ser condición de posibilidad y objetivo final del cambio organizacional? Quizás la esencia misma de la Organización Inteligente esté encapsulada en esta nueva actitud o nuevo espiritu. Tratemos de penetrar, entonces, el significado de esta misteriosa actítud.

\section{La esencia del cambio organizacional impulsado por la Quinta Disciplina}

En referencia a la actitud que se alcanza practicando la disciplina del "Dominio Personal" (Senge, 1990: 183), nos dice lo siguiente:

"Las gentes con alto nivel de dominio personal comparten varias caracteristicas. Tienen un sentido especial de propósito que subyace a sus visiones y metas. Para esas personas, una visión es una vocación y no sólo una buena idea. [...] Han aprendido a percibir las fuerzas del cambio y a trabajar con ellas en vez de resistirlas. Son profundamente inquisitivas, y desean ver la realidad con creciente precisión. Se sienten conectadas con otras personas y con la vida misma. [...] Se sienten parte de un proceso creativo más amplio, en el cual pueden influir sin controlarlo unilateraimente" (subrayado mio).

Dos de los temas aquí presentes parecen ser los mismos del "pensamiento sistémico": una percepción aguda de la realidad y la constatación de que la acción humana es más efectiva en colectividad. Pero junto a estos temas aparece otro, ligado a las visiones y a la vaga noción de "vocación". Más adelante Senge dirá también que el "Dominio Personal" es: "[...] un proceso de focalizarse continuamente, una y otra vez, en lo que uno desea de veras, en nuestras visiones."

El punto empieza a aclararse cuando sale a flote el tema de la felicidad humana. Senge dice: "[...] Nadie puede demostrar ni refutar la afirmación de que los seres humanos tienen un propósito. [...] Pero como premisa funcional, la idea tiene una gran potencia. Entre otras cosas, implica que la dicha puede ser un resultado de vivir en coherencia con nuestro propósito" (Senge, 1990: 191, subrayado mío).

Senge (1990: 356) confirma esta idea cuando habla de la dicha lograda mediante la libertad; pero no cualquier forma de libertad, sino mediante la "libertad para' (en contraste con la 'libertad respecto de') es la libertad para crear los resultados que de veras deseamos. Es la libertad que buscan las personas que procuran el dominio personal. Es el corazón de la organización inteligente, porque el impulso hacia el aprendizaje generativo es el deseo de crear algo nuevo, algo que tenga valor y significado para la gente."

Lo que Senge está diciendo, en otras palabras, significa: si aceptáramos 
-como "premisa funcional"- que la felicidad (o libertad) del ser humano consiste en realizar acción teleológica efectiva, entonces, al combinar esta idea con el "pensamiento sistémico", obtenemos como consecuencia inmediata que el hombre puede alcanzar una felicldad plena solamente mediante la participación en la acción teleológica colectiva. Esta "acción teleológica colectiva" no es otra cosa, por supuesto, que una Organización.

Esta parece ser, pues, la esencia de esa nueva actitud (o espíritu) que constituye, simultáneamente, la condición de posibilidad y el resultado de la aplicación de las 5 disciplinas: es la búsqueda de la felicidad mediante el volcamiento de todo nuestro ser hacia la persecución efectiva del fin organizacional. Es condición de posibilidad porque está basada sólo en una "premisa funcional" -por tanto no hay ninguna seguridad de que sea realmente válida para todos. Se requiere, entonces, como mínimo, una "opción" inicial por esa actitud para poder aplicar las 5 disciplinas. Es resultado porque, precisamente, la construcción de esa actitud en todos los miembros de la organización es lo que persigue la Quinta Disciplina: esta actitud crearía un profundo compromiso con el fin organizacional que, a su vez, se traduciría en una maximización de la eficiencia en el funcionamiento de la organización. Como podemos ver ahora, asi quedarían resueltos magistralmente los tres problemas que se propone solucionar la corriente del nuevo humanismo. Veamos, detenidamente, en qué consiste esta solución.

\section{La clave de la solución: La nueva} relación individuo-organización

La "gran potencia" que tiene la "premisa funcional" antes citada es que abre la posibilidad de un relación completamente nueva, mutuamente provechosa, entre el individuo y su organización. Si el individuo descubre que, al comprometerse con la organización, esta se vuelve el escenario más propicio para su autorrealización, la organización confiadamente podrá abrirse más a una participación activa por su parte en la toma de decisiones. Pero, igualmente, sólo si en la organización se da esa apertura, sus miembros podrán percibir como deseable y atractivo el involucrarse y comprometerse con ella. El logro de este fin constituiría una perfecta simbiosis entre el individuo y la organización. Simbiosis que, evidentemente, resuelve los tres problemas mencionados en la Introducción. Esta simbiosis se construye en torno a un "pacto" (en contraposición a la noción de "contrato"), entre el individuo y la organización:

"[...]'En el tipo de organización que procuramos construir, el pleno desarrollo de las personas está en el mismo plano que el éxito financiero. [..]' Ver el desarrollo de la gente como un medio para los fines de la organización desvaloriza la relación que puede haber entre el individuo y la organización. [...] Una relación completa requiere de un pacto... una relación de pacto descansa sobre un compromiso compartido con ideas, problemas, valores, metas y procesos de administración... Los pactos reflejan unidad, gracia y equilibrio. Expresan la indole sagrada de las 
relaciones" (Senge, 1990: 186, subrayado mio).

La posibilidad de vivir la experiencia de un "pacto" -forjado en base a fines comunes que permitan el "compromiso"es, también, causa de que, en general, cambie la actitud del individuo hacia su trabajo. En las organizaciones tradicionales el "contrato" implicaba la idea de una necesidad de intercambio de bienes entre las partes -indispensable para alcanzar, cada una por su cuenta, sus fines particulares. El trabajo era, por tanto, sólo una obligación contraída, un medio necesario para desempeñarse en la vida extra-organizacional. En las nuevas organizaciones, en cambio, la idea del "pacto" y del "compromiso" borra las diferencias entre lo intra-organizacional y lo extra-organizacional: todo queda subordinado a la visión organizacional compartida, a sus fines y valores. Tal es el caso, por ejemplo, de la relación trabajo-familia, tratado explícitamente por Senge (1990: 384386):

"[...] El viejo mundo de límites precisos entre el trabajo y la familia se está des. moronando. [...] En la organización inteligente, los limites entre lo personal y lo organizacional se difuminan intencionalmente. [...] El conflicto entre el trabajo y la familia se reduce abruptamente cuando la organización impulsa valores acordes con los sentimientos de la gente, valores que tengan igual significación en el trabajo y en el hogar. Sólo entonces será posible que los ejecutivos dejen de vivir ateniéndose a dos códigos de conducta y comien- cen a ser una sola persona" (subrayado mío).

Por todo lo dicho anteriormente, la Quinta Disciplina aparece no como una simple tecnología de gerencia más, sino como un discurso portador de un proyecto social. La visión de futuro -el "nuevo mundo" que la Quinta Disciplina dibuja ante nosotros- es la de una sociedad completamente organizada en términos de "organizaciones", sin intersticios extra-organizacionales, donde los individuos actúan siempre de acuerdo con el mismo "código de conducta" y se autorrealizan descubriendo nuevos modos de lograr efectivamente los fines de las organizaciones en las que participan. En fin, es un mundo en el que reina la paz y la armonía y el bienestar entre los hombres.

\section{El proyecto social de la modernidad}

Las ideas de "humanismo", "libertad", "felicidad", "bienestar" que aparecen en el discurso de Senge, junto con la observación de que aparentemente la Quinta Disciplina es portadora de un proyecto social, hace surgir inmediatamente la pregunta: ¿es la Quinta Disciplina una inconsciente impulsadora, o rescatadora, de la utopia social de la era moderna lanzada en los tiempos de la llustración? En otras palabras, ¿es un humanismo ilustrado el nuevo humanismo de la Quinta Disciplina? Para dar respuesta a estas cuestiones debemos examinar primero en qué consiste el proyecto social de la Modernidad. Acudamos, entonces, a una de las figuras filosóficas más excelsas 
que contribuyeron, en el siglo XVIII, con la formulación de tal proyecto: Immanuel Kant ${ }^{1}$.

\section{La dimensión moral y política de la libertad}

La "libertad" y la "dignidad" del ser humano, son, en efecto, los temas centrales de la Modernidad desde sus mismos origenes en el siglo XVIII. Sin embargo, éstos no son dos elementos independientes que, reunidos, producen el discurso moral y político moderno. Más bien, la libertad y la dignidad humanas aparecen como dos caras de una misma moneda que podríamos llamar "moralidad racional". En efecto, la libertad es pensada por Kant como la capacidad que posee el ser humano de guiar racionalmente su propia conducta, sin atender a lo que le es dictado o impuesto "desde afuera" de su propia racionalidad. La libertad, entendida en ese sentido, no es otra cosa que autonomía. Pero, ¿cómo es posible la autonomía en el hombre?

Según Kant (1785), la autonomía sólo puede descansar en la facultad de la Razón de ser práctica por sí sola, es decir, en la facultad que tiene la Razón de producir por si misma principios para la acción y producir por sí misma un interés en guiarse por tales principios. Esto trae dos importantes consecuencias. La primera es que estos principios se le presentan al ser humano como incondicionalmente necesarios. La segunda es que, dado que la Razón es Razón Univer- sal, estos principios se presentan como absolutamente universales, válidos para todo ser racional. Estos principios racionales constituyen, por tanto, una moral racional que es pensada, simultáneamente, como el último fundamento ontológico de todas las moralidades existentes en las sociedades humanas.

Ahora bien; ya hemos visto que la libertad es pensada, modernamente, como la facultad del ser humano de guiarse por una moral racional. Pero, ¿cómo entra el tema de la "dignidad" humana en todo esto? La "dignidad" es pensada como un concepto generado por la misma moral racional. La moral racional, tal como lo muestra Kant, gira en torno al respeto a la naturaleza racional como un "fin en si mismo". La naturaleza racional es un fin en sí mismo en el sentido de que está predestinada para la realización plena de su racionalidad -que no es otra cosa que la autonomía. De manera que la moral racional "privilegia" al hombre, como ser racional, por encima de todas las demás cosas. Esta condición privilegiada del ser humano constituye, precisamente, su "dignidad". Vemos, entonces, cómo la libertad, entendida como autonomía, es, simultáneamente, la fuente de la dignidad humana y la fuente de la necesidad de respetar tal dignidad: el respeto a la racionalidad y la autonomía del otro constituye el ejercicio de mi propia racionalidad y autonomía.

Kant (1797) entiende que de este respeto al otro nace la necesidad de una

1 Para un resumen más detallado del pensamiento moral y político kantiano, consúltese a Suárez (1997). 
unión civil bajo leyes comunes garantizadas por un ente con el poder y la autoridad suficientes: el Estado. En el estado pre-civil, o estado de naturaleza, nadie está seguro de las acciones de los demás, todos hacen lo que les parece correcto y el daño a la humanidad no puede ser impedido ni produce consecuencia alguna. La razón de ser del Estado radica, entonces, en hacer efectiva la justicia, es decir, hacer efectivo el respeto a la dignidad humana. Desde esta perspectiva el Estado luce como un "representante" de la Razón: aquel ente que traduce los principios morales racionales en leyes jurídicas y ejerce el poder necesario para hacerlas efectivas. De este sentido del Estado -como autoridad jurídica última que decide acerca de lo correcto y lo incorrecto- se desprende la obligación moral de respetar sus leyes a toda costa.

Ahora bien; ¿qué consecuencias trae esta visión moral y política moderna de la sociedad para el modo como cada individuo establece sus relaciones con los demás agentes sociales, en particular, con las organizaciones?

\section{De la libertad al uso "público}

\section{y "privado" de la Razón}

Hay que destacar, ante todo, que la función central del Estado, tal como éste es pensado por Kant, es la de definir y hacer respetar los derechos humanos -que no son otra cosa que privilegios asociados a la dignidad de la condición humana. Un ingrediente importante de estos derechos es la garantía de un espacio de holgura en el que el individuo pueda desenvolverse desembarazadamente. Porque sólo al disponer de tal holgura el individuo puede abordar la tarea de construirse a sí mismo en torno a su destino esencial: la racionalidad. Es en este espacio de holgura, definido y garantizado por el Estado, donde los individuos llevan a cabo sus empresas, es decir, se abocan a la realización de los propósitos que ellos tienen. Es también aqui donde establecen contratos privados entre ellos para así poder lograr con mayor facilidad estas empresas. Pero, ¿cómo compaginan los individuos sus fines particulares con el fin universal que tienen como seres humanos?

Kant (1784) aclara el asunto estableciendo una división entre el uso "público" y el uso "privado" de la razón. De acuerdo con esta división, los individuos de una sociedad moderna se desenvuelven en dos dominios de racionalidad claramente distintos. El uso "público" de la razón es aquel que el individuo hace en su condición de ciudadano, es decir, como miembro de una comunidad politica cuyo propósito es potenciar la humanidad o racionalidad de todos sus miembros. El ciudadano se dirige a sus pares para ofrecer el fruto de sus reflexiones acerca de la adecuación o inadecuación de las leyes que rigen al colectivo social. Por el contrario, el uso "privado" de la razón es aquel que el individuo hace en su condición de empleado de una organización, o, dicho de manera más general, como instrumento al servicio de unos ciertos fines privados. En ese caso el individuo pone su razón al servicio de unos fines particulares, ya sean los suyos propios o los de otro.

Ambos dominios resultan de fundamental importancia en la sociedad. El uso público de la razón permite impulsar el progreso político de la sociedad como un 
todo. Pero el uso privado permite que se lleven a cabo una serie de tareas circunstanciales que constituyen la infraestructura organizativa que sostiene a la sociedad. Nótese sin embargo, que el uso público de la razón es jerárquicamente superior con respecto al privado. Esto debido a que la condición de ciudadano es más propia de la dignidad humana que la condición de mero empleado o instrumento. Las consideraciones políticas propias del ciudadano acercan al ser humano a aquella universalidad racional que es fuente de la autonomía. Y sólo gracias a que el individuo se entiende a sí mismo como ciudadano de una comunidad política, está dispuesto a ponerse como instrumento al servicio de tareas particulares que contribuyen con tal comunidad.

Ahora estamos preparados para dar respuesta a la pregunta de en qué medida el proyecto social implícito en la "Quinta Disciplina" constituye un rescate del proyecto social de la Modernidad que acabamos de resumir.

\section{El carácter no-moderno del proyecto social de la "Quinta Disciplina"}

Podemos distinguir, en principio, tres diferencias fundamentales entre la visión moderna de la sociedad y la visión codificada en el discurso de Senge. En primer lugar, notamos una diferencia a nivel de la noción misma de "libertad". En segundo lugar, notamos una diferencia en lo referente a la distinción "público" vs. "privado". En tercer lugar, notamos una diferencia en lo referente a la relación individuo-organización. Como veremos a continuación, desarrollando en detalle las tres diferencias lograremos mostrar que todas ellas están estrechamente vinculadas entre si.

\section{La noción de "libertad"}

En lo que respecta a la noción de "libertad", es evidente que para Senge esta palabra significa la posibilidad de realizar las aspiraciones personales que cada individuo tiene. Estas aspiraciones, como ya hemos visto, se funden con los fines de la organización en la que trabaja el individuo, con lo cual la libertad queda transiormada en la posibilidad de realizar los fines organizacionales. La libertad, por tanto, implica aquí un compromiso con ciertos fines organizacionales circunstanciales y no con una idea de racionalidad universal. $A$ diferencia del hombre modemo, que se debe exclusivamente a la Razón Universal, el hombre trazado por la "Quinta Disciplina" se debe exclusivamente a la Organización Particular.

Queda, sin embargo, un cierto rastro de aquella idea de universalidad asociada a la razón. Senge afirma, en efecto, que la aspiración más propia del ser humano es aprender. El "aprendizaje" parece elevarse así a la condición de aquella naturaleza humana universal asociada a la razón que predicaban los filósofos de la llustración. Pero nótese que la noción de "aprendizaje" se refiere sólo a la capacidad para lograr con efectividad lo que se desea. Esto implica que la aspiración universal del ser humano es realizar sus fines particulares, cualesquiera que ellos sean. De aquí que el rastro de universalidad que queda en este discurso funciona, en el fondo, como un negador de cualquier idea universal acerca de los propó- 
sitos esenciales del ser humano. Pareciera que aquí ocurre una extraña inversión o hasta confusión entre los fines universales del ser humano y sus fines particulares. Esto nos lleva a la segunda diferencia, la referente a la distinción entre uso público y uso privado de la razón.

\section{El uso público y el uso privado de la razón}

Lo primero que debemos notar es que, en términos de esta distinción, la "Quinta Disciplina" parece ubicar la libertad humana en el ámbito del uso privado de la razón, es decir, exactamente al contrario de como to hacía la llustración. En efecto, como hemos dicho, en el proyecto social de la Modernidad el uso público de la razón es superior con respecto al uso privado, debido a que su tema es el bien universal. Es en el ámbito del uso público de la razón donde el individuo descubre los principios morales que le permiten ser autónomo, es decir, libre. En cambio, según la "Quinta Disciplina", el hombre despliega su libertad persiguiendo con máxima efectividad los fines de la organización de la cual es miembro. El ámbito organizacional obviamente corresponde al uso privado de la razón, donde lo que se persigue no es necesariamente un fin universal, de validez objetiva, sino un fin circunstancial, válido sólo subjetivamente.

Poniendo lo anterior en otras palabras: frente al hombre moderno que aspiraba a elevarse por encima de su propósito privados para alcanzar su destino y su libertad en la realización de un fin universal, encontramos a un hombre "sengeano" que proclama que su destino y su libertad están en la realización de sus propósitos privados. Pero esta afirmación no es del todo exacta, pues ella sugiere que la división ilustrada entre uso público y uso privado de la razón tiene validez para la "Quinta Disciplina". Sin embargo, si examinamos de nuevo el discurso de Senge notamos, en primer lugar, que resulta imposible distinguir en él un espacio que corresponda al ámbito del uso público de la razón. Pareciera que, según la "Quinta Disciplina", todo uso de la razón es privado. Pero nótese que, en ausencia de un ámbito "público", carece de sentido hablar de un ámbito "privado". Esto debido a que estas dos nociones se definen por mutua oposición: lo público es lo que no es privado y lo privado es lo que no es público. Lo que parece ocurrir, en el fondo, es que la "Quinta Disciplina" hace que desaparezca la misma distinción entre lo público lo privado. $O$, dicho de otro modo, el ámbito organizacional pasa a asumir ambos roles simultáneamente. $Y$ esto nos lleva, finalmente, a la tercera diferencia.

\section{Individuo y organización}

¿Cómo es posible el solapamiento de las funciones del ámbito público y el privado en el espacio organizacional? Como ya hemos dicho, en el pensamiento filosófico y politico de la llustración la vida social de un individuo transcurre o bien en el ámbito privado o bien en el ámbito público. Esto permite que el individuo entienda a la organización en la que labora como sólo una parte de la totalidad de su vida social. Más allá de la organización está el ámbito público, donde ocurre la realización de la humanidad esencial de cada quien. Nótese que bajo esta configuración resulta diff́cil concebir de qué modo el individuo puede comprometerse tal radicalmente con el fin organizacional 
como lo pretende Senge. Esto, debido a la existencia de un ámbito extra-organizacional que es mucho más importante que el intra-organizacional. De acuerdo con esto último, entonces, pareciera que, para que ocurra una fusión entre los fines del individuo y los de su organización, hace falta, primero, que el ámbito público ya no pueda hacerse espacio fuera de las organizaciones.

Esto es, en efecto, lo que parece perseguir explícitamente la "Quinta Disciplina" al abogar por una sociedad completamente colmada de organizaciones, sin intersticios inter-organizacionales. Al desaparecer totalmente estos intersticios, el ámbito público no tiene más remedio que alojarse en el interior de las organizaciones, haciéndose privado, mientras que el ámbito privado no tiene más remedio que hacerse público. En ambos movimientos las diferencias entre los dos ámbitos tienden a diluirse por completo. Pero, ¿mediante qué mecanismo puede ocurrir tal dilución entre dos elementos que, originalmente, son opuestos? Sólo es necesaria una pequeña transformación de aquello en torno a 10 cual gira el ámbito público: la noción de libertad. Basta con definir esta palabra en términos propios del ámbito privado, 0 , mejor aún, en términos del ámbito privado organizacional, para que ocurra esta sorprendente síntesis entre ambos elementos en la organización. Como vemos, esto nos refiere, de nuevo, a la primera di- ferencia, la establecida en torno a la noción de libertad.

\section{Observación final}

En la última sección hemos recorrido tres diferencias fundamentales existentes entre la "Quinta Disciplina" y el pro. yecto social de la Modernidad. La libertad definida ya no en torno a fines universales sino particulares, la desaparición de la diferencia entre el ámbito privado y el público, la desaparición de los intersticios inter-organizacionales: estos son tres elementos propios de la "Quinta Disciplina" que se definen y fundan mutuamente. La pregunta que queda abierta, entonces, es por aquello que subyace de fondo a través de este sistema de diferencias. ¿De dónde surge la intención que opera tal transformación sobre el proyecto social de la Modernidad, apropiándose de su lenguaje pero modificando completamente sus significados claves? ${ }^{2}$ Sólo respondiendo a esta pregunta será posible entender más a fondo cuál es el sentido de las tecnologías gerenciales contemporáneas. Tal reflexión se hace aún más urgente en vista de la gran influencia que estas tecnologias empiezan a ejercer en una multitud de ámbitos de la vida social. Evadir esta pregunta es evadir pensar acerca de las fuerzas a las que estamos siendo sometidos a diario de manera invisible pero constante.

2 Una línea de indagación paralela a la presentada en este artículo puede ser encontrada en Suárez (1998), donde se intenta interpretar las ideas de la "Quinta Disciplina" como un retorno al modo de pensamiento propio de la Antigüedad. 


\section{Agradecimientos}

Deseo expresar mi agradecimiento al Prof. Jorge Dávila por haberme introducido al tema de las "Tecnologías Gerenciales" a través de un curso del mismo nombre dictado por él en el marco del Postgrado en Sistemología Interpretativa de la Universidad de Los Andes.

\section{Bibliografía citada}

Deming, W. E. (1993). The New Economics for Industry, Government, Education, Massachusetts Institute of Technology Center for Advanced Engineering Study, Cambridge MA.

Hammer, M. \& Champy, J. (1993) Reengineering The Corporation, Harper Business.

Jackson, M. (1993). Beyond The Fads: Systems Thinking for Managers. University of Hull. Working Paper No. 3.
Kant, E. (1784). “¿Qué es la llustración?". Publicado en Filosofía de la Historia (pag. 25-38). Fondo de Cultura Económica. México. 1981.

Kant, E. (1785). Fundamentación de la Metafísica de las Costumbres, Colección Austral, España.

Kant, E. (1797). Metafísica de las Costumbres, Tecnos, España.

Majima, I. (1994). The Shift to JIT: How People Make the Difference, Productivity Press, Portland, USA.

Senge, P. (1990). La Quinta Disciplina. Cómo impulsar el aprendizaje en la organización inteligente, Ediciones Juan Granica, España.

Suárez, R. (1997). "El carácter problemático de la situación penitenciaria venezolana". Frónesis, Vol 4, N². Universidad del Zulia.

Suárez, R. (1998). "An inquiry into the Historical Meaning of 'The Fifth Discipline'". Artículo aceptado en Systems Practice and Action Research. University of Hull. 